livraisons

d'Histoire

de l'Architecture

\section{Livraisons de l'histoire de l'architecture}

$36 \mid 2018$

Jean-Louis Pascal (2)

\title{
Les expositions universelles, Jean-Louis Pascal, critique et constructeur
}

The world exhibitions, Jean-Louis Pascal, a critic and a builder

Weltausstellungen. Jean-Louis Pascal als Kritiker und Gestalter

\section{Anne Richard-Bazire}

\section{(2) OpenEdition}

Journals

Édition électronique

URL : http://journals.openedition.org//ha/1019

DOI : $10.4000 /$ /ha. 1019

ISSN : $1960-5994$

Éditeur

Association Livraisons d'histoire de l'architecture - LHA

Édition imprimée

Date de publication : 15 décembre 2018

Pagination : 11-29

ISSN : 1627-4970

\section{Référence électronique}

Anne Richard-Bazire, "Les expositions universelles, Jean-Louis Pascal, critique et constructeur », Livraisons de l'histoire de l'architecture [En ligne], 36 | 2018, mis en ligne le 15 décembre 2020, consulté le 25 janvier 2021. URL : http://journals.openedition.org/lha/1019 ; DOl : https://doi.org/10.4000/lha. 1019 


\section{LES EXPOSITIONS UNIVERSELLES, JEAN-LOUIS PASCAL, CRITIQUE ET CONSTRUCTEUR}

L'architecte parisien Jean-Louis Pascal $^{1}$ (1837-1920) a mené de front tout au long de sa vie d'architecte de nombreuses activités: critique, constructeur, chef d'atelier à l'École des Beaux-Arts, membre de l'Institut, du conseil des bâtiments civils et de plusieurs sociétés pour lesquelles il travaillera très activement jusqu'à sa mort. Il participe à de nombreuses reprises aux expositions universelles, évènements majeurs du XIX ${ }^{\mathrm{e}}$ siècle, vitrines des progrès techniques et des réalisations industrielles de nations voulant témoigner de leur dynamisme économique. Critique reconnu de ses pairs, Jean-Louis Pascal est choisi comme rapporteur des expositions universelles de 1873 à Vienne et de 1900 à Paris en tant que membre du jury de la section d'architecture. C'est l'occasion pour lui de prendre position sur l'éclectisme architectural et l'internationalisation des styles qu'il voit poindre au tournant du siècle. En 1878, Jean-Louis Pascal est l'architecte de la section portugaise de l'exposition universelle qui se tient à Paris. Sa reconstitution très réussie du monastère des Hiéronymites de Belem lui vaut une médaille de première classe ${ }^{2}$ et l'honneur d'être décoré Chevalier de l'Ordre de Santiago du Portugal.

\section{L'exposition universelle de 1873 à Vienne}

En 1873, Pascal est rapporteur de l'exposition universelle de Vienne à la demande de César Daly. "N'ayant pas d'architecture à faire, [écrit-il à Charles Garnier] je ne suis pas le moins du monde fâché de me rabattre sur la littérature. Il [Daly] m'offre deux cents francs pour chaque feuille d'impression ${ }^{3}$. " Il consacre donc un très long rapport ${ }^{4}$ dans la Revue Générale de l'Architecture à l'architecture autrichienne mais aussi française, russe (ill. 1), etc. L'exposition qui se déroulait dans le parc du Prater (ill. 2), à Vienne, était organisée sous la direction architecturale du viennois Karl Freiherr von Hasenauer $^{5}$ (1833-1894). Elle couvrait une

1. Pour en savoir plus sur ses années de jeunesse et sa formation, voir Anne Richard-Bazire, "JeanLouis Pascal ", Livraisons d'histoire de l'architecture, $\mathrm{n}^{\mathrm{o}}$ 28, Paris, Chirat, $2^{\mathrm{e}}$ semestre 2014, $226 \mathrm{p}$.

2. "Récompenses de l'exposition universelle de 1878. Grands prix et médailles ", R.G.A.T.P., $\mathrm{n}^{\circ} \mathrm{XXXV}$, 1878, p. 235.

3. Bibliothèque de l'École des Beaux-Arts (B.E.B.A.), Ms 744, Lettres de l'architecte Jean-Louis Pascal à Charles Garnier, lettre no 50, 7 septembre 1873.

4. Jean-Louis Pascal, "Exposition universelle de Vienne ", R.G.A.T.P., n ${ }^{\circ}$ XXX, 1873, p. $204-209$ et p. 251-259. R.G.A.T.P., n ${ }^{\circ}$ XXXI, 1874, p. 22-35, p. 56-65, p. 265-274. R.G.A.T.P., n ${ }^{\circ}$ XXXI, 1874, p. 99-110, p. 193-202.

5. On lui avait adjoint trois architectes : Gugitz, Corompay et Rumpelmayer. 


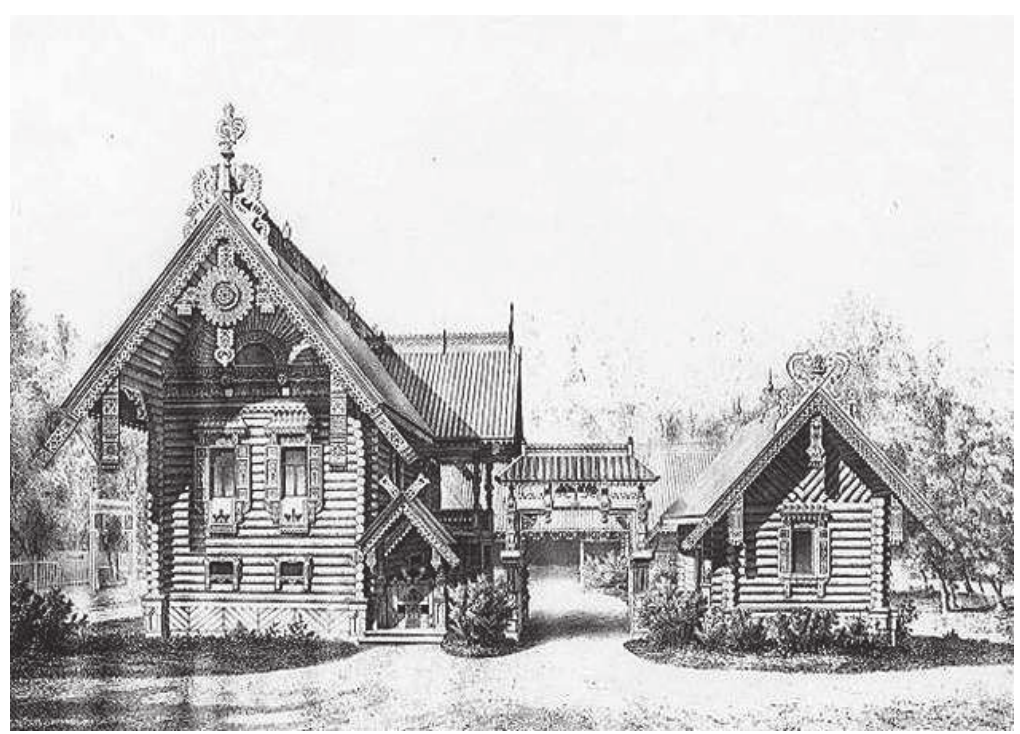

Ill. 1 : Exposition universelle de Vienne, 1873. Habitations des paysans russes. Jean-Louis Pascal, "Exposition universelle de Vienne. L'exposition d'architecture ", R.G.A.T.P., nº XXXI, 1874, p. 32.

superficie de $1984000 \mathrm{~m}^{2}$ et exposait 50000 concurrents $^{6}$. Dans le plan primitif ${ }^{7}$ en arête de poisson choisi pour les bâtiments par le directeur de l'exposition, de Schwarz-Senborn, les nationalités se succédaient d'ouest en est, de l'Amérique du nord au Japon. En dehors des constructions disséminées dans le parc (jouant le rôle de la galerie d'alimentation de l'exposition de Paris en 1867), et de quelques bâtiments administratifs, il y avait cinq grands bâtiments principaux. Deux servaient à l'exposition d'agriculture. Le palais de l'Industrie, dont une immense rotonde de cent huit mètres de diamètre constituait le centre ${ }^{8}$, couvrait sept hectares; c'était une longue halle centrale coupée en son milieu par la rotonde, arête principale sur laquelle venaient se greffer perpendiculairement les salles affectées aux nations. La galerie des machines qui occupait, sur quatre hectares, un long bâtiment, s'étendait parallèlement à ce bâtiment principal, sur huit cents mètres. À

6. Vingt-deux ans auparavant, l'exposition de Londres couvrait une surface de $96181 \mathrm{~m}^{2}$ et accueillait 17000 concurrents.

7. Disposition défigurée dans le plan définitif par les additions successives de bâtiments dans les espaces libres qu'on destinait au jardin. Jean-Louis Pascal regrette que l'on ait abandonné le plan ingénieux choisi par l'architecte Krantz pour l'exposition universelle de Paris en 1867. La division par nationalité s'imposait ; on lui adjoignit un rangement par ordre de matière, ce qui permettait de comparer les produits similaires de tous les pays tout en étudiant l'ensemble de chaque nationalité. C'était l'adoption de la table à double entrée en usage en statistique et dans d'autres sciences. "On réalisait, avec le meilleur mode d'appréciation, la philosophie même de l'Exposition ". JeanLouis Pascal, "Exposition Universelle de Vienne ", R.G.A.T.P., n XXXI, 1874, p. 101.

8. Jean-Louis Pascal considérait comme une prouesse architecturale d'avoir couvert un espace d'une telle portée. L'idée première de couvrir d'une énorme tente un cercle de $108 \mathrm{~m}$ de diamètre venait d'un ingénieur anglais, Scott Russel; la direction des travaux fut confiée à l'ingénieur en chef Schmidt et l'exécution à la maison Harkort, de Duisbourg. La hauteur totale de l'édifice était de $84 \mathrm{~m}$. Le toit conique reposait sur 32 colonnes de fer de $24,40 \mathrm{~m}$ de hauteur, posées sur une fondation en béton. Le diamètre de la grosse lanterne était de $32,40 \mathrm{~m}$, celui de la petite de $8 \mathrm{~m}$. 


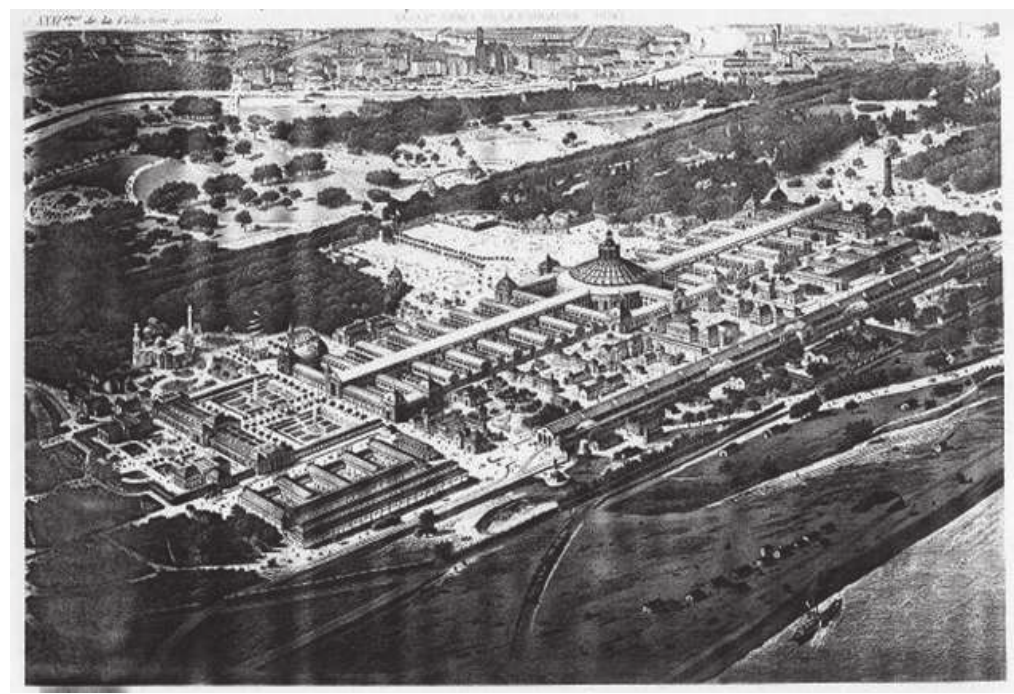

Ill. 2 : Exposition universelle de Vienne, 1873. Vue d'ensemble de l'exposition dans le parc du Prater. Jean-Louis Pascal, "Exposition universelle de Vienne. L'exposition d'architecture ", R.G.A.T.P., $\mathrm{n}^{\circ}$ XXXI, 1874, p. 25.

l'extrémité est de la grande galerie se dressait, perpendiculairement, le bâtiment de l'exposition des Beaux-Arts. Les projets architecturaux exposés dans cette partie sous forme de dessins, pouvant d'ailleurs être hors concours, permirent à JeanLouis Pascal d'étudier les écoles des divers pays, leur vitalité, et, s'agissant de constructions réalisées, de caractériser l'architecture des pays exposés.

Après avoir fait quelques considérations générales sur l'architecture autrichienne et sur Vienne, Jean-Louis Pascal commente la création de la Ringstrasse", " la réalisation la plus rapide et la plus grande opération de construction qu'aucune ville ait pu oser dans ce siècle ${ }^{10}$ :

«Partout sur les boulevards neufs, aux larges trottoirs plantés, aux amples chaussées, de hautes et vastes constructions, épaisses de murs, grandes d'échelle, fortes de saillies, avec des avant-corps, des corniches puissantes, des colonnes dégagées, des balcons saillants, de grosses chaînes, de riches chambranles, des ors, des sculptures. Tout cela présente un aspect de fermeté et de grandeur dont aucune de nos rues neuves à Paris ne saurait donner l'idée. Cette grandeur n'exclut pas une certaine monotonie; mais c'est là un point dont nous aurions, nous autres Parisiens, mauvaise grâce à faire la critique, car je ne sais rien de déplorablement écœurant comme ces longues enfilades de fenêtres pareilles qui sont maintenant le caractère de nos grandes voies urbaines [...] je dirais même que le premier aspect est plus

9. Le 20 décembre 1857, l'empereur François-Joseph $1^{\text {er }}$ (1830-1916) signait le décret décidant du démantèlement des remparts qui enfermaient le centre-ville de Vienne. Il créa ensuite un boulevard annulaire longé de bâtiments publics et de maisons de rapport, la Ringstrasse, un des plus beaux boulevards de la capitale autrichienne. Celui-ci délimite le $1^{\text {er }}$ arrondissement qui correspond au centre historique de la ville, sur la rive droite du Danube.

10. Jean-Louis Pascal, "Exposition universelle de Vienne », R.G.A.T.P., n ${ }^{\circ} \mathrm{XXX}, 1873$, p. 206. 
beau à Vienne, puisqu'il est plus grand, plus imposant [...] Certes, leur art à eux n'est pas d'une délicatesse épurée; c'est peut-être plus du savoir que de l'art ${ }^{11}$.

Dans la maison à loyer, l'esthétique prime sur le confort :

"La maison à loyer prend extérieurement l'aspect d'une grande habitation [...] Tant pis, par exemple, si les commodités d'intérieur, dont on nous a tant gâtés à Paris, ne sont pas satisfaites [...] On pousse le scrupule des façades jusqu'à proscrire les tuyaux de descente. Il faut que l'aspect extérieur soit satisfait [...] Force maisons particulières et édifices privés sont l'œuvre des architectes les plus marquants du pays, tandis qu'on cite à Paris comme des exceptions les habitations privées exécutées par nos maîtres ${ }^{12}$.

Pascal attribue ce résultat au fait qu'en Autriche, la situation faite à l'architecte est fort différente : c'est, à Vienne, un artiste déchargé de la responsabilité matérielle de ses œuvres, car il est toujours doublé d'un constructeur. Il n'en conserve que la responsabilité artistique. Et, contrairement à Paris, il existe une tolérance beaucoup plus grande dans la réglementation des saillies, des alignements et des hauteurs ${ }^{13}$.

Mais, pour ce partisan de la vérité constructive, un "vice capital bien grave " affecte l'habitat privé : si toutes les maisons ont l'air d'être en pierre, c'est «un affreux ciment que chacun colore à son gré, ou plutôt, comme il peut, [qui] reproduit toutes les formes qu'on veut lui infliger " ${ }^{14}$.

«De robustes consoles vont porter en avant les fortes dalles des balcons [...] ne soyez pas choqués qu'elles soient en zinc et renferment les extrémités de solives en fer qui remplissent la véritable fonction de supports. Un bel enduit ou une bonne couche par-dessus raccordera tout cela [...] Dans tous ces murs de face il n'y a que briques, et l'on n'en voit pas une seule [...] Aussi arrive-t-on à ce résultat étrange, qu'une ville à qui l'utilisation des matériaux qu'elle trouve abondamment, ménageait les ressources d'une originalité toute particulière, présentera dans quelques années le plus bel exemple qu'on puisse voir de banalité grandiose ${ }^{15}$.

Plus loin Pascal écrit : "À Vienne, comme ailleurs, le gâchis le plus complet, le plus savant, fleurit sous le nom d'éclectisme " ${ }^{16}$. Pour donner, malgré le désordre esthétique qui y préside, une idée du formidable effort architectural et urbanistique, tenté depuis quinze ou vingt ans à Vienne, Pascal cite quelques-uns des bâti-

\section{Ibid.}

12. Ibid., p. 207.

13. "La manie de la réglementation excessive n'aura pas peu contribué à gâter notre Paris, par cette uniformité assommante qui nous est en quelque sorte imposée ». Ibid., p. 208.

14. Ibid., p. 208.

15. Ibid.

16. Ibid., p. 252. 


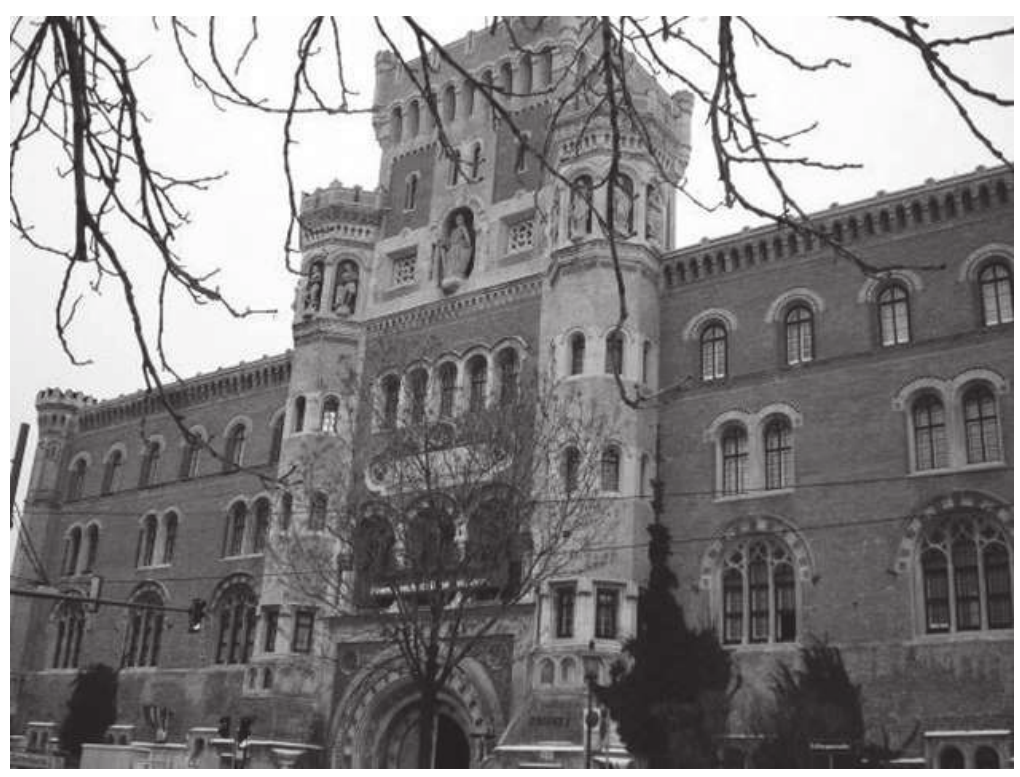

Ill. 3 : L'Arsenal. Façade principale. Architectes Eduard van der Nüll et August von Siccardsburg. Après 1848. 18 Ghegastrasse, Vienne. Cl. auteur.

ments publics viennois : l'Arsenal ${ }^{17}$ (ill. 3), construit après 1848 par les architectes

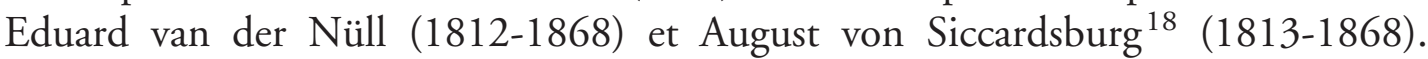
Deux musées destinés à recevoir les collections d'art (le Kunsthistorisches Museum) et d'histoire naturelle (le Naturhistorisches Museum), élevés parallèlement, entre 1871 et 1890, par les architectes Gottfried Semper (1803-1879) et Karl von Hasenauer (1933-1894), sur les deux côtés d'une vaste place (ill. 4 et 5) (le troisième côté étant constitué par les Écuries de la cour), devaient être reliés au Palais impérial nouveau ${ }^{19}$, la Neue Burg, par des arcs de triomphe jetés au-dessus de la Ringstrasse, qui ne furent jamais construits. La Neue Burg devait comprendre deux ailes en arc de cercle rajoutées à l'arrière du château ancien ${ }^{20}$. Le Palais de l'Université, construit par l'architecte Heinrich von Ferstel (1828-1883), était destiné à réunir en un seul gigantesque bâtiment tout l'enseignement universitaire : huit cours, des salles de fêtes, des bibliothèques, faculté de médecine, de droit, de théologie et de philosophie, archives, musées, salles de cours. La Paradeplatz verra s'élever le Palais de Justice, le Parlement, inspiré de l'architecture antique, construit par l'architecte danois Theophile Hansen (1813-1891) et l'Hôtel de ville, immense monument de $19000 \mathrm{~m}^{2}$ élevé par l'architecte Friedrich von Schmidt (1825-1891), à partir de 1872, en style gothique. La construction d'églises, de théâtres, d'opéras,

17. L'Arsenal est situé au $n^{\circ} 18$ de la Ghegastrasse à Vienne.

18. Architectes de l'Opéra de Vienne (1861-1868), le Staatsoper, qui ouvre le 25 mai 1869 avec une représentation du Don Giovanni de Mozart. Pratiquement détruit en 1945, il est reconstruit à l'identique et rouvre ses portes le 5 novembre 1955.

19. Euvre gigantesque constituant un ensemble analogue au Louvre et aux Tuileries, réunissant et embellissant des corps de bâtiments disparates et accumulés d'un premier palais : la Hofburg, transformation due aux architectes viennois Hasenauer, élève de Van der Nüll et Siccardsburg, et allemand Semper.

20. Une seule aile sera ajoutée. La Neue Burg abrite la Bibliothèque nationale et plusieurs musées. 


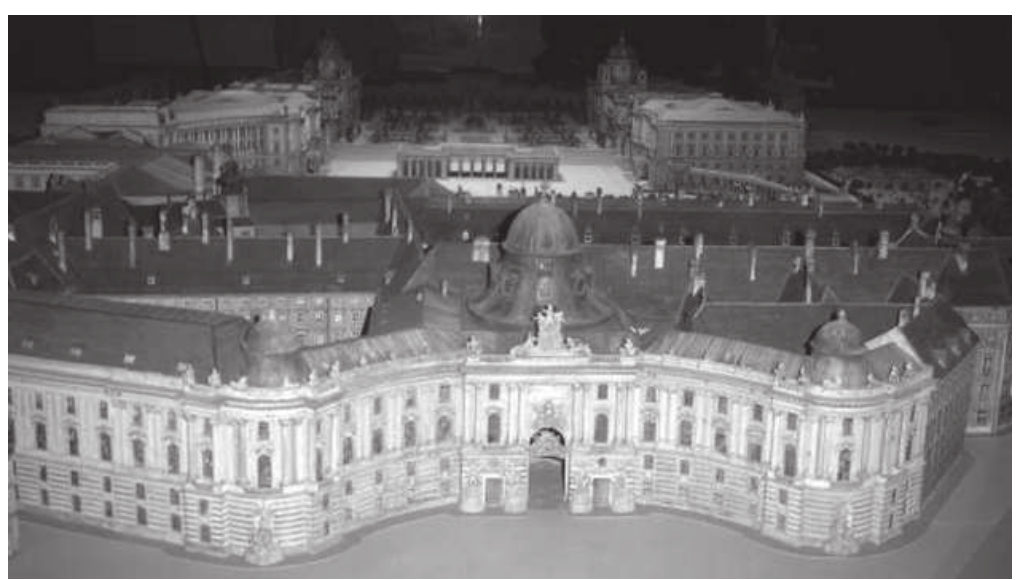

Ill. 4 : Maquette de l'extension du palais de la Hofburg. Vienne. Vers 1900. La maquette représente l'œuvre gigantesque réunissant et embellissant des corps de bâtiments disparates et accumulés d'un premier palais : la Hofburg (au $1^{\text {er }}$ plan) et de nouveaux bâtiments : la Neueburg (bâtiment en arc de cercle situé sur la gauche en arrière du palais de la Hofburg), et les Kunsthistorisches Museum et Naturhistorisches Museum, situés de part et d'autre de la place nouvelle (tout au fond), transformation due aux architectes viennois Karl von Hasenauer et allemand Gottfried Semper. Palais de la Hofburg, Vienne. Cl. auteur.

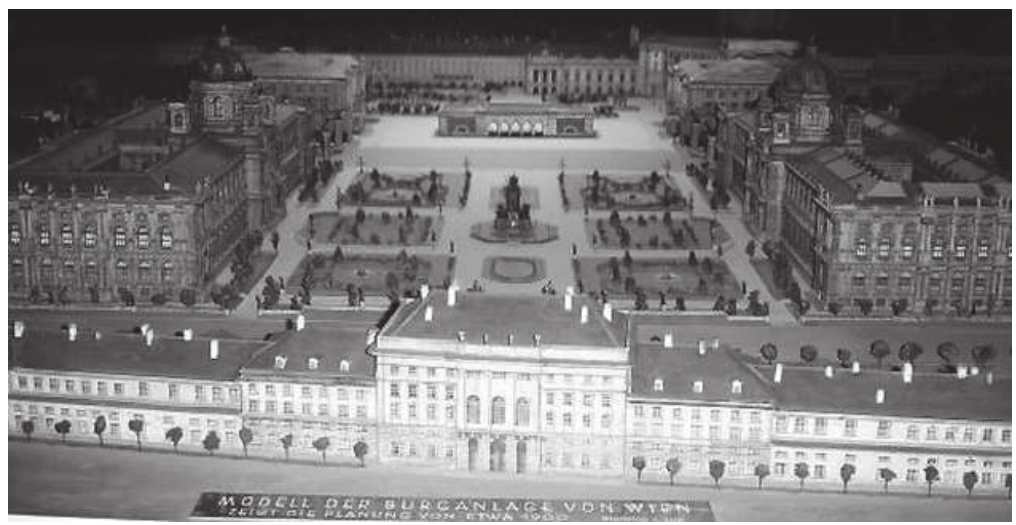

Ill. 5 : Maquette de l'extension du palais de la Hofburg. Vue de l'autre côté. Vienne. Vers 1900. La maquette représente les Kunsthistorisches Museum et Naturhistorisches Museum, situés de part et d'autre de la nouvelle place, et le palais ancien de la Hofburg tout au fond, transformation due aux architectes viennois Karl von Hasenauer et allemand Gottfried Semper. Palais de la Hofburg, Vienne. Cl. auteur.

[Les deux photos représentent la même maquette vue des deux points opposés].

d'écoles, de gares, de halles, d'aqueducs pour l'alimentation en eau, de ponts sur la Wien et le Danube, de parcs, comme le Prater, « la plus grande promenade dont une ville puisse s'enorgueillir", redessiné par un ingénieur français, Gondouin, témoignent de ce fantastique mouvement viennois: "Nous devrions assister à l'éclosion d'une grande école ${ }^{21}$, d'une grande manifestation du génie humain, car

21. "La suite [de l'article] nous montrera combien peu d'Autrichiens figurent dans cette pléiade d'artistes à qui fut confiée la réfection de la capitale : si une école sérieuse s'y constitue dans l'avenir, elle aura dû entièrement son origine à des étrangers». Jean-Louis Pascal, "Exposition universelle de Vienne. L'exposition d'architecture », R.G.A.T.P., vol. XXXI, 1874, p. 25. 
jamais plus belle occasion ne s'est produite, pour les artistes, de trouver la gloire et de laisser des œuvres, que dans cette rapide et gigantesque réfection d'une ville entière $"^{22}$.

Le nombre d'œuvres exposées par les Autrichiens à l'exposition est par conséquent considérable; aucune autre ville ne peut présenter un ensemble d'édifices commencés aussi important, pas même la ville de Paris. Il y a très peu de dessins théoriques, très peu de restaurations, encore moins d'œuvres d'études. C'est l'impulsion donnée aux travaux qui a forcé à la construction d'une nouvelle école et entraîné sa réorganisation. Car il n'y avait pas de traditions en Autriche ; l'enseignement était peu important ; il n'existait pas de fondations favorisant les études, les voyages, les travaux théoriques de longue haleine. Des efforts individuels seuls ont pu produire quelques résultats, et c'est à l'étranger que l'on a trouvé des hommes capables de répondre à un développement si inattendu de l'architecture : "L'exposition viennoise n'est pas, à proprement parler, autrichienne " ${ }^{23}$. JeanLouis Pascal trouve en revanche, à l'exposition des ouvres russes, les traces d'un enseignement académique, d'une école sérieuse dont la plus haute récompense est une bourse de voyage permettant aux artistes d'étudier l'Italie, la France, la Grèce, l'Espagne et l'Orient. Mais en Russie, contrairement à l'Autriche, les éléments de production ne sont pas au niveau des éléments d'études. Dans les œuvres bâties, connues par le dessin ou la photographie, "deux courants fort contraires se produisent : l'architecture nationale, indigène, traditionnelle, et l'inspiration de l'éclectisme d'Occident " ${ }^{24}$. En général, toutes ces œuvres sont des édifices privés, les travaux exécutés pour l'État russe, les villes ou les grandes administrations étant rares. Le plus haut niveau d'études est offert par l'Italie, à travers l'exposition, par ailleurs trop sommaire, de "travaux théoriques", "travaux de restauration ", et "travaux modernes ». Jean-Louis Pascal note à propos de l'Italie: "Il y a plaisir à voir pratiquer si naturellement [...] la décentralisation : Milan, Naples, Florence, Rome, Bologne et les petites villes même sont des centres d'art bien vivants, que nous souhaitons ne pas voir se fondre dans l'unification italienne ${ }^{25}$. L'Allemagne est sous représentée, numériquement et artistiquement : "Il ressort d'une façon très évidente que l'École de Berlin l'emporte sur l'enseignement de Munich ; mais, dans les deux expositions, les monuments remarquables sont peu nombreux, les études théoriques ne sont nullement représentées, les restaurations archéologiques pas davantage ; le pastiche règne en maître ${ }^{26}$. La peinture, en revanche, a fait des progrès immenses en Allemagne et "son exposition luttait d'intérêt et d'importance numérique avec la nôtre " ${ }^{27}$. La Belgique "dont l'école de peinture tient un rang

22. Jean-Louis Pascal, "Exposition universelle de Vienne ", R.G.A.T.P., no XXX, 1873, p. 258.

23. Jean-Louis Pascal, "Exposition universelle de Vienne", R.G.A.T.P., no XXXI, 1874, p. 35.

24. Ibid., p. 63. Citons par exemple les œuvres de M. H. Bosse représentant par la photographie, car les plans font défaut, comme pour la plupart des architectes russes, une "Maison de campagne du comte Schuwaloff", près de Saint Pétersbourg, en style rococo ; une Villa du grand-duc Michel en style Renaissance italienne, une Chapelle funéraire du comte Kotschubey en style russe.

25. Jean-Louis Pascal, "Exposition universelle de Vienne ", R.G.A.T.P., n XXXI, 1874, p. 270.

26. Ibid., p. 268.

27. Ibid., p. 265, 267. 
si élevé " ${ }^{28}$, expose peu d'architecture. L'Espagne n'est guère mieux représentée : "La plupart des compositions y sont médiocres, dans un misérable style gothique, ou dans ce style classique qui florissait chez nous à l'époque de la Restauration " ${ }^{29}$, alors que son exposition de peinture est très brillante. Jean-Louis Pascal s'attarde peu sur la Grèce, dont "les monuments modernes sont, pour la plupart, exécutés par des danois ou des allemands ", le Danemark qui fournit des architectes à l'étranger et dont "les peintres et sculpteurs ont des ouvres remarquables à l'Exposition ", et le Portugal qui "n'existe pas pour l'architecture, du moins à Vienne " ${ }^{30}$.

Rapportant cette fois sur la section française, Pascal écrit :

«Ici, contrairement à ce que nous avons vu en Autriche, l'enseignement, l'École, les traditions jouent un grand rôle; l'intervention de l'État dans l'entretien de nos monuments historiques a contribué à former une école archéologique dont nous ne voyons l'équivalent nulle part ailleurs. L'École de Rome et la direction de ses travaux forment un grand courant d'autres études théoriques [...] Pour le reste des productions françaises, c'est-à-dire pour les créations modernes, je crois avoir employé, dans mon étude antérieure sur le monument architectural à Vienne, le mot gâchis, en le rapprochant d'éclectisme, et je le laisserai dans sa trivialité, parce qu'il me semble exprimer franchement les tentatives divergentes de toutes nos individualités. Elles sont bien de leur temps, sans en avoir conscience : choisissant surtout telle voie parce qu'elle n'est pas frayée, abandonnant la route simple parce que la curiosité et l'attrait de la recherche les poussent vers l'inconnu, visant à l'effet par le bizarre et l'imprévu, touchant à tout, contrôlant tout, avec cet esprit de libre examen et de mépris des notions admises, qui en art comme en science, comme en politique, comme en philosophie, a eu jusqu'ici l'apparence du désordre, mais pourrait bien devenir, une fois réglé, la marque caractéristique de notre temps, le véritable esprit scientifique. ${ }^{31}{ }^{\prime}$

\section{L'exposition universelle de 1878 à Paris}

En 1878, Pascal s'embarque pour le Portugal qui l'a choisi comme architecte des galeries portugaises qui s'insèreront dans la grande rue des Nations du palais du Champ de Mars de l'exposition universelle de 1878, à Paris. Il y passe une semaine, se repérant grâce au guide Lavigne de la collection Joanne, et à celui de John Murray "toujours si précieusement fourni de renseignements de toute sorte " ${ }^{32}$. Il rédige une longue "Lettre de Lisbonne" dans la Revue Générale de l'Architecture $^{33}$ :

28. Ibid., p. 272.

29. Ibid.

30. Ibid., p. 273.

31. Ibid., p. 60.

32. Germond de Lavigne, Itinéraire de l'Espagne et du Portugal, Paris, Librairie Hachette et John Murray, Handbook for travellers in Portugalo, London, Albemarle street.

33. Jean-Louis Pascal, «Lettre de Lisbonne ", R.G.A.T.P., $\mathrm{n}^{\circ} \mathrm{XXXV}$, 1878, p. 24-30, 67-74, 164-168. 
«Le tremblement de terre de 1755 a tout détruit à Lisbonne et n'a laissé subsister que de bien rares débris des édifices anciens ; aussi faut-il accoutumer ses yeux à voir une ville entière, dont toutes les parties qui ne sont pas absolument modernes datent du siècle dernier, présenter les spécimens du rococo le plus mouvementé et le plus portugais, aussi bien dans son architecture religieuse que dans son architecture civile. Les constructions récentes exécutées par des artistes indigènes, témoin l'Hôtel de Ville en cours de construction, rappelleraient même encore cette influence [...] Mais les étrangers ont pénétré ici plusieurs fois, par voie de choix ou de concours et, l'un des monuments décoratifs les plus considérables élevés de notre temps à Lisbonne, la colonne triomphale à Don Pedro IV, est due à la collaboration de MM. Davioud et Elias Robert " ${ }^{34}$.

Le mode de construction utilisé, plus de cent vingt ans après le désastre, trahit encore les craintes d'un tremblement de terre; on monte tous les planchers des étages sur des pans de bois couronnés d'un comble, avant d'élever les murs contre lesquels les poteaux sont adossés, sans en être solidaires. Les murs ne servent qu'à protéger des intempéries et de l'indiscrétion. L'emploi du fer est d'ailleurs très rare dans la construction, car on l'importe d'Angleterre ou de France, ce qui en rend l'usage très onéreux. Jean-Louis Pascal est vivement intéressé par un mode décoratif très particulier au Portugal, les faïences, qui ornent les palais mais aussi les maisons particulières, même les plus modestes. Il va d'ailleurs rapporter de son voyage une assez grande quantité de spécimens de faïences historiques :

"Outre des fragments franchement moresques ou directement inspirés de l'art arabe, il n'est pas rare de trouver de riches spécimens de la Renaissance, dans lesquels de beaux jaunes et des blancs éclatants viennent se mêler à la couleur toujours dominante, le bleu, qui vaut à ces terres cuites le nom d'azulejos " ${ }^{35}$.

À l'exposition universelle de 1878, la section portugaise était disposée le long de la rue des Nations, mitoyenne de la section du Luxembourg. Venait ensuite une voie de cinq mètres de large, reliant la rue des Nations aux jardins, qui la séparait de la section des Pays-Bas. Cette disposition permit à Jean-Louis Pascal d'exposer sur trois côtés une série d'arcades, reliées à la reproduction du porche (ill. 6 et 7) de l'église du monastère des Hiéronymites de Belem près de Lisbonne, qui constituait le clou et la façade de l'exposition portugaise. À son arrivée au Portugal, en pénétrant par l'embouchure du Tage, l'architecte décrit le monument :

"Tous les voyageurs avaient remarqué au passage la grosse tour carrée de Belem, à peu de distance de laquelle s'étend, parallèlement à la rive, l'église du même nom, abritant un cloître fameux, spécimen remarquable de l'art portugais au $\mathrm{XVI}^{\mathrm{e}}$ siècle, du temps de ce grand roi Emmanuel (Manoel) [...] qui a donné son nom à un style éminemment indigène. C'est bien, en effet, un art particulier, où l'influence de l'Italie et de l'Espagne se fait évidemment sentir " ${ }^{36}$.

34. Ibid., p. 25-26.

35. Ibid., p. 27.

36. "On attribue le couvent de Belem (de Bethléem) à un italien nommé Potassi, c'est un spécimen remarquable de l'art portugais au XVI ${ }^{\mathrm{e}}$ siècle, de style emmanuelin ». Jean-Louis Pascal, «Lettre de Lisbonne ", R.G.A.T.P., 1878, p. 25. 


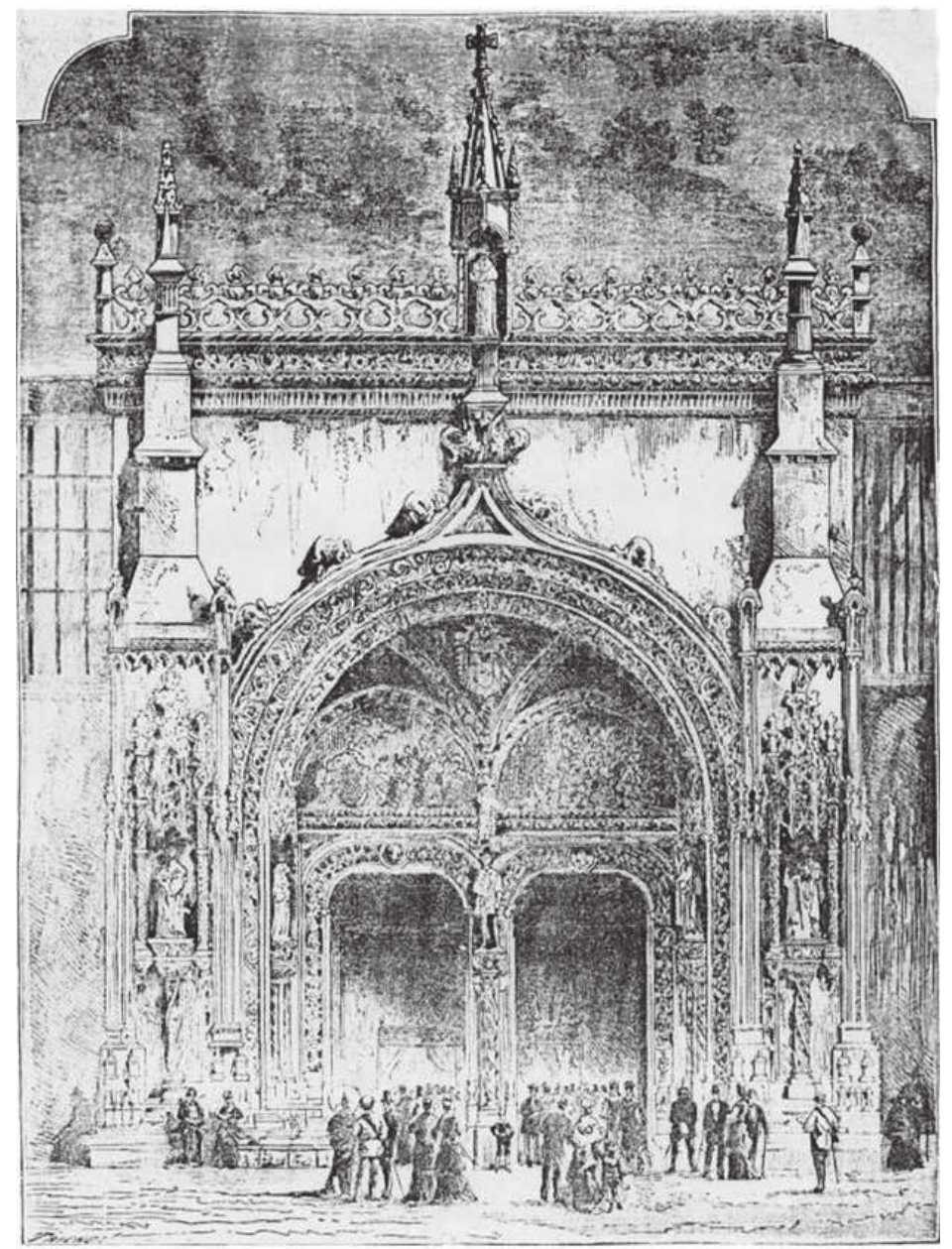

Ill. 6 : Façade portugaise de l'exposition universelle de 1878. Architecte Jean-Louis Pascal. Sculpteurs : Antoine Watrinelle et Gustave Germain. Reproduction du porche de l'église du monastère des Hiéronymites de Belem au Portugal. XVI ${ }^{\mathrm{e}}$ siècle. Architectes João de Castilho et Diego de Torralva. G.B.A., 1879 , p. 246.

Pour cette reconstitution, Jean-Louis Pascal diminua les proportions, tronqua la partie supérieure de la façade de l'église et reconstitua les motifs au moyen de croquis et de photographies pris par lui sur les lieux. Son idée première avait été d'employer, pour la décoration de toute sa construction, des reproductions fidèles, des estampes, qui auraient été d'une mise en plan facile, et dont le caractère archaïque eût été indiscutable. L'Académie des Beaux-Arts de Lisbonne lui avait d'ailleurs gracieusement envoyé tout ce qu'elle possédait comme estampages et fragments d'architecture et de sculpture d'art portugais. Mais les estampages n'avaient pu être utilisés à cause de la différence d'échelle, le manque de temps empêchant ensuite Pascal de mettre son projet à exécution. Pour la façade, comme pour les arcades du cloître, aucun ornement, aucun bas-relief, n'avait été moulé sur les originaux. "C'est donc à Paris que tout a été fait: l'étude, la composition et les modèles " ${ }^{37}$. L'architecte remplaça les images des saints patrons de l'édifice, qui

37. A. Dupuis, "La section portugaise. Arcades intérieures et pavillon des colonies ", La Semaine des constructeurs, 1878-1879, p. 78-79. 


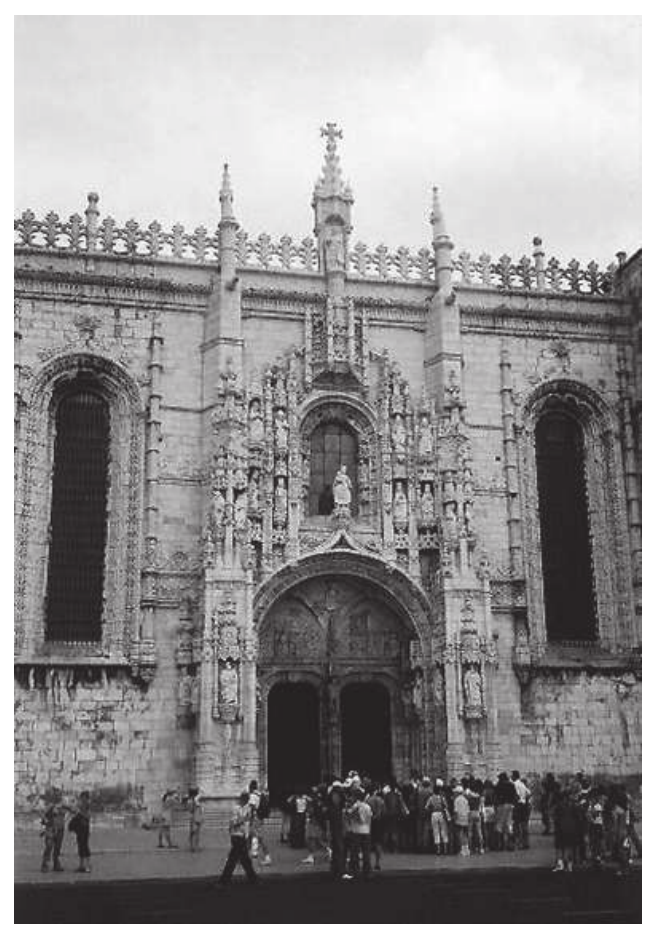

Ill. 7 : Porche de l'église du monastère des Hiéronymites de Belem au Portugal. XVI ${ }^{\mathrm{e}}$ siècle. Architectes João de Castilho et Diego de Torralva. Cl. auteur.

garnissaient les niches du porche de l'église de Belem, par les statues des grands hommes du Portugal, à l'exception des statues de la Vierge et de l'infant don Henrique, couronnant le trumeau du milieu, qui étaient des reproductions aussi approchées que possible des originaux ${ }^{38}$ :

«M. Pascal a restitué, pour la gloire de l'Exposition portugaise, le porche de l'église du fameux monastère des Hiéronymites de Belem près de Lisbonne. Cette architecture, gothique dans sa masse, renaissance dans ses détails, un peu surchargée comme l'art espagnol qu'elle avoisine, mais gardant encore quelque parfum de l'art arabe si longtemps dominateur dans le pays, cette architecture, malgré tant d'influences diverses, reste très particulièrement accentuée par certaines dispositions décoratives qui lui sont propres. Cette restitution est si parfaite de style et de caractère, non seulement dans l'ensemble, mais encore dans les moindres parties, qu'on pourrait la croire moulée sur nature ${ }^{39}$.

Venait, à la suite de la façade de l'église de Belem, une série d'arcades qui formait, sur trois côtés, une sorte de clôture à l'exposition du Portugal. Le premier groupe d'arcades comprenait quatre motifs répétés, empruntés au cloître du monastère de Belem (ill. 8 et 9); pour le deuxième groupe d'arcades, Jean-Louis Pascal

38. A. Dupuis, "La façade portugaise au Champ de Mars ", La Semaine des constructeurs, 1878-1879, p. 66-67.

39. "Exposition universelle de 1878 ", Gazette des beaux-arts, 1879, p. 246. 


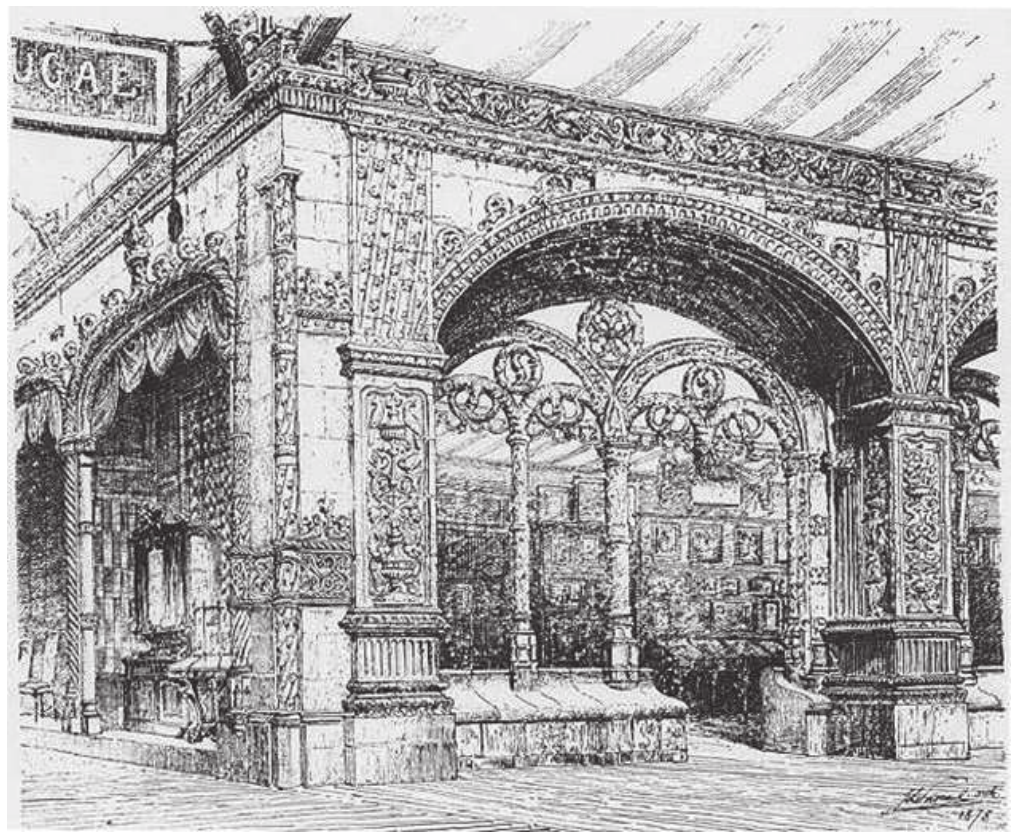

Ill. 8 : Reproduction du cloître du monastère des Hiéronymites de Belem au Portugal, par l'architecte Jean-Louis Pascal et les sculpteurs Antoine Watrinelle et Gustave Germain pour l'exposition universelle de 1878. G.B.A., 1879, p. 246.

avait copié des travées du cloître gothique de l'abbaye de Batalha, visitée lors de son périple au Portugal. De belles reproductions photographiques des deux cloîtres, d'un praticien amateur Relvas, était présentées à l'exposition. Pascal avait été très impressionné par le couvent de Batalha ${ }^{40}$ :

"J'ai fait une courte mais remarquable épreuve en me rendant au couvent de Batalha, le plus important édifice de ce genre, sinon par sa dimension, du moins par sa richesse et son intérêt, parmi ceux qui comptent encore au nombre des curiosités du Portugal [...] C'est d'abord une haute église gothique, dont le style un peu sec rappelle plutôt l'architecture allemande ou anglaise, une grande abside à demi démolie [...] des murailles de couvent et enfin une porte donnant accès au cloître entourant une cour plantée, de vaste proportion. C'est la partie la plus originale de l'édifice. Le gothique raide, déjà signalé à l'extérieur, s'engraisse et se développe dans les claustras à jour qui ferment à demi les arcades. C'est une ornementation toute particulière, remarquablement ferme et dessinée, qui ne rappelle aucune disposition du même genre dans notre pays; elle marque la transition entre la fin du Moyen Âge et le style d'Emmanuel, dont le plus beau spécimen est au couvent de Belem. La photographie en a maintenant répandu les motifs ; j'ai tâché d'en reproduire le plus simple (car ils sont fort variés) dans une partie de l'exposition portugaise, au Champ de Mars; il forme deux des arcades de la clôture des galeries intérieures de la section ${ }^{41}$.

40. L'abbaye, dont le nom signifie : "abbaye de la bataille " a été fondée par le roi Joao $1^{\text {er }}$ à l'endroit d'une de ses victoires. Le roi Manuel voulait faire de ce lieu destiné à sa sépulture, l'endroit le plus riche de la péninsule.

41. Jean-Louis Pascal, "Lettre de Lisbonne », R.G.A.T.P., n XXXV, 1878, p. 165-166. 


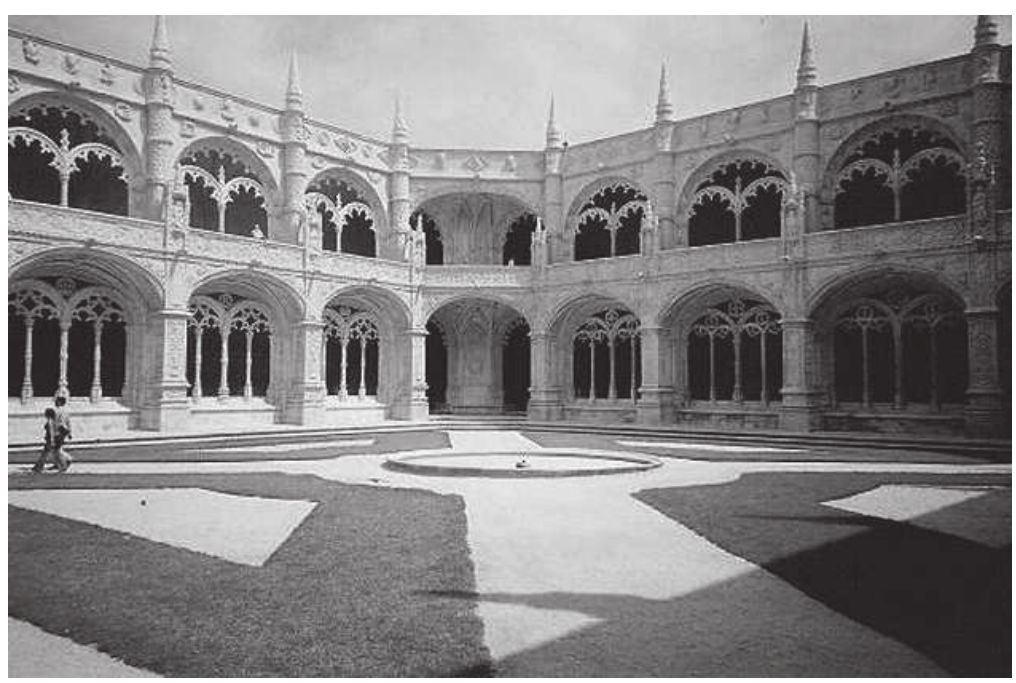

Ill. 9 : Cloître du monastère des Hiéronymites de Belem au Portugal. XVI ${ }^{\mathrm{e}}$ siècle. Architectes João de Castilho et Diego de Torralva. Cl. auteur.

Le troisième groupe d'arcades, ainsi que les façades en retour sur les galeries longitudinales, étaient des motifs de fantaisie inspirés de l'architecture locale et surtout de l'abbaye de Belem.

Deux sculpteurs parisiens, Antoine Watrinelle (1818-1913) et Gustave Germain (1843-1909) ${ }^{42}$, ont réalisé «la masse d'ornements qui couvre cette architecture bizarre " ${ }^{43}$, ainsi que celle des façades du pavillon des colonies, que Jean-Louis Pascal construit également pour la section portugaise ${ }^{44}$.

Celui-ci était placé extérieurement dans les jardins. Il était remarquable surtout par la reproduction qui y était faite de faïences décoratives. Tous les dessins de céramique du pavillon étaient des imitations d'originaux relevés sur place par Pascal, pendant son voyage, ou prêtés par l'Académie des Beaux-Arts de Lisbonne. Ils avaient été peints sur toile et vernis, de façon à donner l'illusion de la couleur et du brillant de la faïence. La couverture du pavillon, en zinc peint et verni, repro-

42. Ils avaient créé la "Société en nom collectif Watrinelle et Germain " en 1869 ; elle fut dissoute en 1881 après 24 chantiers menés en commun. Michel Gasse $(\dagger)$, qui avait travaillé sur la biographie de Gustave Germain, m’avait donné très aimablement ces renseignements.

43. A. Dupuis, "La section portugaise. Arcades intérieures et pavillon des colonies ", La Semaine des constructeurs, op. cit., p. 79.

44. Il y eut deux marchés passés entre les deux sculpteurs ornemanistes et Pascal, représentant provisoirement le gouvernement portugais. Le premier date du 25 octobre 1877 et concerne les travaux de sculpture des intérieurs de l'exposition universelle, section Portugal, composés de motifs de Batalha et de Belem, d'une façade formant la troisième travée ainsi que des retours sur les galeries longitudinales. Celles-ci devaient être commencées le 5 décembre 1877 et terminées le 5 février 1878, sous peine d'un dédit de $50 \mathrm{fr}$. par jour de retard. Les sculpteurs étaient payés $21250 \mathrm{fr}$. Le second marché date du 20 décembre 1877 ; il concerne les travaux de sculpture de la façade nationale et des façades du pavillon des colonies. Les sculptures devaient être exécutées et posées entre le $1^{\text {er }}$ février et le $1^{\text {er }}$ avril 1878 , à peine d'un dédit de 50 fr. par jour de retard. Les sculpteurs étaient payés $11000 \mathrm{fr}$. pour la façade nationale et $5960 \mathrm{fr}$. pour le pavillon. Ces contrats m’ont été aimablement communiqués par les descendants de Gustave Germain. 
duisait une couverture en tuiles émaillées disposées en écailles, telles qu'on les employait autrefois ${ }^{45}$. Les formes générales du pavillon étaient empruntées à l'architecture portugaise. Celui-ci se composait d'un petit porche percé de trois baies en façade et d'une vaste salle rectangulaire surmontée d'un dôme. Toute la construction était en bois. L'intérieur était recouvert de planches en sapin, le parement extérieur était fait d'un lattis enduit de plâtre, sur lequel on avait marouflé des toiles peintes. Sur la façade, étaient inscrits les noms des principales colonies portugaises ${ }^{46}$. Les vitraux, très simples, décorés des armes du Portugal, avaient été apportés de Lisbonne. Pascal fut loué pour la rigueur de son travail : "L'exposition du Portugal est, on le sent tout de suite, l'œuvre d'un artiste consciencieux qui, disposant de peu de place, d'un budget restreint et d'un temps très court, est parvenu néanmoins à imprimer un excellent caractère à l'exposition portugaise " ${ }^{47}$. En remerciement, les architectes portugais participeront à la souscription ouverte en 1898 pour ériger, à côté de l'Opéra de Paris, un monument à Charles Garnier, monument réalisé par Jean-Louis Pascal ${ }^{48}$.

\section{Le critique du mouvement architectural contemporain, miroir de l'époque}

Un an après l'Exposition universelle de 1878, au moment du décès de Louis Duc, Jean-Louis Pascal livre à la Gazette des beaux-arts ses réflexions sur l'architecture française qui lui semble présenter malgré les différentes époques qui se sont succédées et qui, chacune, ont marqué de leur style leur génération, une grande unité. "Ce qui frappe le plus dans la hauteur de son talent [dit Pascal de Louis Duc], c'est bien cette transmission d'un esprit très français, fait de justesse et de grandeur ${ }^{49}$ :

«L'architecture est de tous les arts celui qui, par son essence et les conditions spéciales suivant lesquelles il s'exerce, se prête le moins à ces fluctuations rapides de la mode, qui seront peut-être la caractéristique la plus marquée des efforts de notre époque troublée [...] Pourtant, si peu variés qu'aient paru être pour notre art depuis le commencement du siècle les mouvements si brusques, si opposés, qui ont marqué, par exemple, les productions de la peinture en France, il n'est pas trop difficile à un oil exercé de reconnaître, même dans l'ensemble des œuvres inférieures semées dans

45. A. Dupuis, "La section portugaise. Arcades intérieures et pavillon des colonies ", La Semaine des constructeurs, op. cit., p. 79.

46. Au-dessus de l'entrée: "Cabo Verde, Angola, S. Thome e Principe»; sur le retour à gauche est inscrit : "India-Macau ", et à droite : "Moçambique ".

47. A. Dupuis, "La section portugaise. Arcades intérieures et pavillon des colonies ", La Semaine des constructeurs, op. cit., p. 79.

48. "Le monument à Charles Garnier ", L'Architecture, 1900, 13e année, no 22, samedi 2 juin 1900, p. 189.

49. Jean-Louis Pascal, "M. Duc et son influence sur le mouvement architectural contemporain ", Gazette des beaux-arts, 1879, I, p. 430-443. 
nos villes agrandies, la marque de l'époque de la Restauration, du règne de Louis-Philippe, et du Second Empire [...] Comme on pourrait dire de l'architecture de Louis XIV que la grandeur qui dominait invinciblement toute cette époque se traduit par la largeur de ses voies, la hauteur de ces étages, l'ampleur de ses formes, indépendamment de son style même " ${ }^{50}$.

Ce qu'Erwin Panofsky ${ }^{51}$ appelait "l'habitus », cet esprit du temps qui imprègne tous les aspects d'une société à un moment donné, John Flaxmann, dans Lectures on Sculpture (1810), l'explique en ces termes :

«De la même manière que nous désignons [par le terme style] les phases successives du progrès et du déclin de l'art, nous renvoyons aussi, indirectement, au progrès de l'esprit humain et aux conditions de la société : car les œuvres correspondent aux inclinaisons de leur temps, et les mains réalisent de préférence les objets sur lesquels l'intelligence et la sensibilité se plaisent à s'arrêter ».

"Telle Société, telle Architecture » : cette formule lapidaire d'un César Daly, héritier de Saint-Simon (1760-1825) ${ }^{52}$ et influencé par les théories d'Auguste Comte (1798-1857) et d'Hippolyte Taine (1828-1893) ${ }^{53}$, Jean-Louis Pascal pourrait la revendiquer, qui voit dans les rapports entre l'art et l'industrie de la deuxième partie du siècle, la marque de la société bourgeoise :

«Et si nos successeurs peuvent reprocher à notre temps de n'avoir pas su faire la part assez grande à l'élément d'art dans l'énorme développement de l'industrie, cette abondance même dans la production, cette maigreur économique, ce soin du détail, les habiletés des arrangements des intérieurs [...] y seront certainement la marque [...] de notre génération en architecture. Il y a peut-être là une sorte d'invasion de la bourgeoisie dans l'art qui ne sera pas sans conformité avec nos tendances dans le domaine de la politique et avec nos transformations sociales, et qui justifiera une fois de plus cette vérité, que l'architecte est un auxiliaire précieux pour l'historien ${ }^{54}$.

Propos mesurés de l'élève de Charles Garnier, qui n’hésitera pas à être beaucoup plus incisif en affirmant : "Si les artistes suivaient la voie où ce vampire qu'on nomme l'art industriel voudrait le mener, c'en serait fait de l'école française " ${ }^{55}$.

50. Ibid., p. 430-431.

51. Erwin Panofsky, Architecture gothique et pensée scolastique, Paris, éd. de Minuit, 1967, 221 p.

52. Sur l'apport des idéologies saint-simonienne et fouriériste à la R.G.A.T.P., voir les développements très intéressants de Marc Saboya dans Presse et architecture au XIX eiècle, Paris, Picard, 1991, p. $127-136$.

53. Ibid., p. 190.

54. Jean-Louis Pascal, "M. Duc et son influence sur le mouvement architectural contemporain ", Gazette des beaux-arts, op. cit., p. 431.

55. Charles Garnier, À travers les arts, précédé de : François Loyer, Les ambiguütés de Charles Garnier, Paris, Picard, 1985, p. 182-183. 
Comme César Daly, Jean-Louis Pascal pense donc que "l'art n'est qu'un miroir qui reflète l'homme et la société contemporaine " ${ }^{56}$. Il pense aussi, qu'au sein même de l'art, les différentes disciplines tissent des liens entre elles. Julien Guadet l'a joliment exprimé à propos de l'architecture et la littérature :

"Les arts plastiques ne doivent pas être littéraires, mais en fait leurs évolutions sont préparées, souvent de loin, par les courants que créent les grands écrivains. À Paris, cherchez bien quel est l'architecte de Saint-Philippedu-Roule, et vous trouverez Jean-Jacques Rousseau ; l'architecte de SainteClothilde, et vous trouverez Chateaubriand " ${ }^{57}$.

La recherche d'horizons lointains, dans le temps puis dans l'espace, qui caractérise, au XIX ${ }^{\mathrm{e}}$ siècle, une partie de la production littéraire et picturale, source de l'éclectisme en architecture, dont Pascal s'était longuement entretenu lors de l'Exposition universelle de Vienne, fut une tentative, parmi d'autres, de rénovation de l'architecture française :

«Parallèlement au mouvement littéraire de la Restauration, suivi du Romantisme, on a vu des maittres dessinateurs en architecture, s'inspirant de ce même esprit chercheur, curieux du passé et du lointain, parfois plus soucieux de l'étrangeté que de la logique, se lancer, appuyés de l'archéologie et des découvertes que les voyages aux pays nouveaux mettaient en lumière, et faire revivre des formes abandonnées, pendant que d'autres créaient tout d'une pièce un art absolument nouveau, sans attache avec rien qui l'eût précédé. Ce serait nous écarter beaucoup de notre sujet que de montrer combien de fois ces tentatives séduisantes, hardies et même productives, à de certains points de vue, mais sans racines dans le sol français, ont pu nuire à la culture logique, normale, naturelle et progressive de notre art. Ça a été l'honneur de ces chercheurs d'ouvrir des voies, de montrer des sources où leurs successeurs iront puiser pour élargir et vivifier le grand courant de notre unité et de nos traditions nationales; mais la plupart de ces pseudoécoles, éclatantes comme des feux d'artifice, se sont épanouies pour disparaître $"^{58}$.

Pour Jean-Louis Pascal, la rénovation du classicisme par une relecture de l'Antiquité, non dans les formes extérieures de son architecture, mais dans celle de sa structure, la compréhension de l'esprit de l'architecture grecque, tel est l'apport des grands architectes du XIX ${ }^{\mathrm{e}}$ siècle. Il rejoint, en cela, Eugène-Emmanuel Viollet-leDuc, qu'il a pourtant beaucoup combattu :

56. César Daly, R.G.A.T.P., no VII, 1847-1848, p. 393, cité dans Marc Saboya, Presse et architecture au XIX siècle, op. cit., p. 190.

57. Julien Guadet, "L'église du Sacré-Cour », La Revue de l'Art ancien et moderne, Paris, 1900, p. 103120, p. 108.

58. Jean-Louis Pascal, "M. Duc et son influence sur le mouvement architectural contemporain ", Gazette des beaux-arts, op. cit., p. 431-432. 
«Depuis la Renaissance, [...] l'architecture de la France s'est rattachée à la grande tradition de l'Antiquité, comme sa législation, comme sa littérature, comme ses mœurs, comme tout ce qui procède de l'esprit latin, dont nous sommes imprégnés par transmission héréditaire [...] Il appartint à notre époque de chercher sous la forme, dans ces grands ensembles auxquels on remontait toujours, la structure avec les moyens de construction, et ce qu'on a appelé le caractère. À mesure que les procédés, les principes si éminemment logiques et d'un goût si raffiné de l'art grec, que son enveloppe si exactement adaptée à la matière mise en œuvre furent mieux connus et plus appréciés, un nouvel enseignement se dégagea de ces grands logiciens et de ces artistes incomparables. Tout un côté de l'art antique, peu compris encore, nous apparut pour former un nouveau point d'attache et un trait d'union plus solide entre le passé et le présent, et surtout pour nous préparer un avenir non encore dégagé [...] c'est le mérite de quelques architectes français, les Gilbert, les Duban, les Labrouste, les Duc, bientôt suivis d'une foule d'artistes doublés d'observateurs, pas tant passionnés d'archéologie qu'on a pu le dire, que dotés par leur époque de cet esprit de recherche et d'investigation [...] de scruter les constructions nouvellement visitées de la Sicile et de la grande Grèce, puis celles d'Athènes même [...] et d'en tirer, avec cette déduction bien simple, mais qui fut le prélude d'une quasirévolution architecturale, que la forme extérieure était et devait être la traduction de la structure intérieure, et que la nature des matériaux employés déterminait des proportions et des rapports essentiellement variés, insuffisamment observés jusqu'alors par les artistes, qui avaient prétendu arrêter et formuler certaines règles fixes d'après les œuvres de l'Antiquité " 59 .

Par cette définition, Pascal se déclare adepte du rationalisme; c'est, sous une autre forme, le principe énoncé par Eugène-Emmanuel Viollet-le-Duc dans son XII Entretien: "Tout emploi dissimulé d'un moyen ne saurait conduire à des formes neuves " ${ }^{60}$. Mais la révolte de 1863 que Pascal avait fomentée, notamment avec Jules Guadet, l'avait montré : la liberté était du côté de l'École des BeauxArts, et non de celui de Viollet-le-Duc et d'une esthétique d'État que ce dernier aurait bien aimé imposer :

"Les principes qu'on y enseigne [à l'École des Beaux-Arts] ne constituent pas une doctrine officielle, les hommes et les institutions qui les mettent en application et les propagent avec l'aide matérielle de l'État s'inscrivent dans un consensus général. Cette architecture, faite d'éclectisme et de rationalité, qui s'impose en France, en Europe et jusqu'au Nouveau Monde, traduit les aspirations de la société toute entière comme l'avait fait en son temps le retour à l'antique ${ }^{61}$.

59. Ibid., p. 433-434.

60. Eugène Emmanuel Viollet-le-Duc, XII Entretien, t. II, p. 67, cité par Jean-Michel Leniaud, Violletle-Duc ou les délires du système, Paris, Mengès, 1994, p. 146.

61. Jean-Michel Leniaud, Viollet-le-Duc ou les délires du système, op. cit., p. 166. 
Pascal est en fait un homme de son temps, un "éclectique raisonnable " ou un " rationaliste tempéré » ${ }^{62}$. Ce mélange d'éclectisme et de rationalité, qui caractérise l'enseignement à l'École des Beaux-Arts de l'époque, est imposé par l'État, parce que ce dernier est devenu le principal donneur d'ouvrages et que «le rationalisme désigne autre chose que ce qu'on a dit jusqu'à présent. Il constitue une réponse aux exigences de l'État moderne qui veut construire beaucoup à faible coût " ${ }^{63}$.

La découverte de la liaison organique de l'élément constructif et de la décoration en architecture avait été, pour Jean-Louis Pascal, capitale :

"Ce fut une Renaissance dans la Renaissance, si ce mot ne peut pas être considéré comme trop prétentieux pour caractériser la rencontre de cette idée modeste qui renferme pourtant tout le germe d'une rénovation fécondée en même temps [...] par l'esprit scientifique. La grande Renaissance nous avait fait voir l'aspect extérieur et l'admirable harmonie des formes trouvées par nos maîtres, les anciens; la résurrection de leurs grandes œuvres avait fait deviner aux artistes les secrets de la création, de la composition - de la théorie de l'architecture, en un mot -, sans laquelle ni construction ni application des formes n'existent et ne valent; elle nous avait fait voir les combinaisons des grandes masses, les effets obtenus par les contrastes et les répétitions; elle avait mis l'imagination aux prises avec la matière. Il semble que cette seconde Renaissance nous a initiés à l'esprit de soin, d'ordre, de détail, de justesse, à la science des maîtres en l'art de construire, et, par cette étude approfondie et détaillée, qu'elle nous ait rapporté, avec l'abondance et la recherche délicate des proportions et des profils qui constituent l'aspect extérieur de l'architecture, l'équivalent de ce qui se poursuivait et se découvrait en même temps en linguistique, en littérature et aussi dans les arts parallèles. Nous avions la mélodie; c'est la science harmonique qui nous fut dévoilée [...] On avait fait beaucoup d'histoire, d'art et de littérature de convention; les travaux modernes visèrent une reconstitution plus probable du milieu social dans lequel les chefs d'œuvre s'étaient produits, et poursuivirent enfin par une méthode plus précise et plus scientifique la vérité, ce critérium de toutes les belles productions " ${ }^{64}$.

Quelques années auparavant, faisant la critique architecturale du Salon, JeanLouis Pascal avait loué le collège Chaptal, réalisé par Eugène Train (1832-1903),

62. Deux qualificatifs employés par Jean-Michel Leniaud, le premier dans Les Bâtisseurs d'avenir. Portraits d'architectes. XIX ${ }^{e}-X X^{e}$ siècle, Paris, Fayard, 1998, p. 166, et le second pour qualifier Julien Guadet, l'alter ego de Jean-Louis Pascal, lui aussi "disciple tempéré de Garnier ». Jean-Michel Leniaud, Viollet-le-Duc ou les délires du système, op. cit., p. 150.

63. Jean-Michel Leniaud, Les Bâtisseurs d'avenir. Portraits d'architectes. XIX $-X X^{e}$ siècle, op. cit., p. 166 et Jean-Michel Leniaud, "Charles Garnier : un oppositore alla centralizzazione stilistica della committenza pubblica ", Arte e Architettura. Le cornici della storia, Bruno Mundadori, Flaminia Bardati et Anna Rosellini éd., 2007, p. 291-304.

64. Jean-Louis Pascal, "M. Duc et son influence sur le mouvement architectural contemporain ", Gazette des beaux-arts, op. cit., p. 434. 
professeur d'architecture à l'École nationale des arts décoratifs, honoré du «Prix Duc », par l'Institut, en 1873 :

"Je place au premier rang le "collège Chaptal", qui n'existe pas seulement sur le papier, mais dont on peut apprécier sur nature, la composition, l'ingéniosité, la recherche, la variété, l'esprit de combinaison, voire même la complication ; car on a toujours un peu les défauts de ses qualités. C'est une des œuvres importantes de ces dernières années dans la voie de la construction décorée, du rationalisme appliqué à notre art, voie qu'a ouverte la génération précédente et où s'est signalé le maître par excellence, M. H. Labrouste " ${ }^{65}$.

Le " prix Duc », ou " Prix des hautes études " avait été créé par son fondateur pour inviter les architectes français «au respect de leur art, à l'admiration de la forme, à la clarté, à la noblesse, à la pureté des lignes et des proportions et au soin amoureux des détails " ${ }^{66}$. C'était le rôle des grands maîtres, selon Pascal, de perpétuer le haut style et la grande école française, "en dépit des tentations auxquelles l'entraînent si facilement l'art facile, la grâce, l'abondance et aussi le goût dispendieux de la redondance et du colifichet $"{ }^{67}$. On note toujours, chez Pascal, la recherche d'une certaine simplicité en architecture, de ce qui convient, le refus des détails inutiles. Ainsi parle-t-il, après avoir pourtant loué le collège Chaptal, « des détails abondamment variés, qui pullulent dans toutes les parties de l'édifice " ${ }^{68}$. C'est le même reproche que l'on trouve sous la plume de Jean-Louis Pascal lorsque, jugeant parmi les envois de Rome de 1877 « une très jolie aquarelle » de la Librairie de la cathédrale de Sienne, il écrit : "M. Lambert nous semble avoir alourdi un peu les détails ravissants de cette salle, aussi harmonieuse que peu timide de coloration. C'est qu'il n'a pas encore perdu tout à fait l'habitude de ces inventions décoratives où il cédait volontiers à l'apparat, au faste, à l'abondance " ${ }^{69}$. Louis Duc laissait un haut enseignement pour les générations futures : "Il leur rappellera périodiquement, si elles s'égarent, qu'il y a au-dessus des caprices de la mode, une grande suite traditionnelle pour notre art, et comme un dépôt, que peu d'artistes sont en état de transmettre et qu'ignore le gros de la foule ${ }^{70}$.

65. Jean-Louis Pascal, "Salon de 1876. Architecture», R.G.A.T.P., no XXXIII, 1876, p. 103-112.

66. Ibid., p. 106.

67. Ibid., p. 107.

68. Ibid., p. 106.

69. Jean-Louis Pascal, "Les Envois de Rome de 1877 ", R.G.A.T.P., n XXXIV, 1877, p. 165-167.

70. Jean-Louis Pascal, "Salon de 1876. Architecture », R.G.A.T.P., op. cit., p. 107. 